\title{
Reestructuración turística y cultura empresarial en Andalucía. Una aproximación cualitativa a la sociología económica de las estrategias empresariales.
}

\author{
Pablo Rodríguez González \\ IESA-CSIC \\ prodriguez@iesa.csic.es
}

Resumen: El presente trabajo recoge el planteamiento y los principales resultados de una investigación sobre los discursos estratégicos de los empresarios y directivos turísticos andaluces. Inspirada por la crítica del actor económico racional de la sociología económica, la perspectiva adoptada indaga en los mecanismos con los que la cultura o ideología de gestión construye o da legitimidad a significados sociopolíticos de lo que constituye la eficacia de una estrategia empresarial. El objetivo del análisis es mostrar cómo el proceso de elaboración de estrategias y, en particular, la elección entre calidad $y$ costes en la reestructuración de los negocios turísticos, ocurre en un marco discursivo articulado por el campo organizacional de los destinos turísticos.

Palabras clave: reestructuración turística, estrategias empresariales, espiritu del capitalismo, cultura empresarial

Abstract: This paper reports the theoretical approach and main results of a research project on the strategic discourses of Andalusia's tourism managers and entrepreneurs. Inspired by economic sociology's criticism of the rational economic actor, we inquire into the mechanisms that the business ideology or culture uses to construct or legitimate sociopolitical meanings of strategic efficiency. The analysis aims to show that strategies are drawn up and decisions about quality and costs in restructuring tourism businesses are made in a discursive frame structured by the organizational field of tourist destinations.

Keywords: Tourism restructuring, business strategies, capitalism spirit, business culture. 


\section{Introducción}

$\mathrm{Al}$ igual que otros muchos sectores productivos, el turismo ha experimentado importantes cambios en su funcionamiento en las últimas décadas debido a la acumulación de cambios tecnológicos (Internet), geopolíticos (globalización) y socioculturales (valores posmaterialistas, consumismo) en las sociedades donde toman forma su oferta (destinos turísticos) y su demanda (países emisores) ( $\mathrm{Hja}$ lager, 2007; Mastny y Peterson, 2001). De esta forma, se señala que el turismo de masas es paulatinamente sustituido por un turismo posmoderno o posfordista (MacCannell, 2003; Urry, 2002), con unos nuevos parámetros de demanda - consumo acelerado de mercancías diversificadas que se diferencian por aspectos intangibles o simbólicos-que reclaman nuevas formas de organización de la oferta (la especialización flexible posfordista) (Ioannides y Debbage, 1997; Lash y Urry, 1998). A estos cambios cualitativos se suma el crecimiento cuantitativo de la oferta turística mundial (OMT, 1997), con una creciente fragmentación del mercado en multitud de destinos y empresas, inmersos en una competencia intensificada en la que entran en juego múltiples ventajas: costes laborales, recursos naturales y culturales, apoyos políticos e institucionales, vínculos con la distribución, reputación, etcétera.

En este contexto, los destinos maduros que, como Andalucía, se desarrollaron según los parámetros del turismo de masas se encuentran en una situación de estancamiento (Butler, 1980) y pérdida de competitividad (Barke y Towner, 2004). Para responder a dicha situación, deben afrontar la reestructuración turística (Agarwal, 2002), un proceso colectivo de cambio organizativo que permita adaptar sus productos, estructuras y modos de funcionamiento a la nueva situación de los mercados turísticos. Dicha reestructuración toma, siguiendo a Porter (1985), una doble dirección: el liderazgo en costes y la diferenciación por la calidad. La primera se basa en la flexibilización y optimización de los procesos productivos, mientras que la segunda se orienta a la especialización y la innovación de productos. Aunque no son estrategias excluyentes, se entenderá que su compatibilidad es limitada: las reducciones de costes pueden acabar mermando la calidad, y la apuesta por la calidad se acaba traduciendo en un crecimiento de los costes. Así mismo, se entenderá que, desde la óptica política de quienes fijan los incentivos al desarrollo turístico en los destinos maduros, la calidad suponga una apuesta más atractiva, ya que se asume que un producto más elaborado requerirá unos inputs (léase trabajadores) más cualificados y mejor remunerados. En una postura que reproducen los directivos hoteleros investigados, la alternativa de la competencia por costes resultaría «suicida», ya que nunca se podrán igualar los costes laborales ni las ventajas fiscales de los países competidores menos desarrollados. 
Llegados a este punto, el objetivo de este trabajo es recoger, de forma sintética, los resultados de una investigación ${ }^{1}$ que buscaba dar cuenta, para el caso concreto de los empresarios y directivos turísticos andaluces, del proceso por el cual se decide qué combinación de calidad y reducción de costes adoptan las empresas como estrategia de reestructuración. A la hora de abordar este proceso no nos hemos centrado en evidencias cuantitativas sobre los factores contextuales o endógenos que pueden llevar, objetivamente, a una población de empresas a decantarse más por una determinada estrategia. Tampoco se trata de un estudio de caso en el que se sigue en el tiempo el cambio organizativo de una empresa. Nuestra atención se dirige al armazón cultural de significados y creencias que los directivos y empresarios, en cuanto grupo social encargado de la elaboración de las estrategias (Mintzberg, 1983; 1988), comparten y utilizan para representarse su experiencia. Nuestra tesis será que las formas locales que adopte esta subcultura empresarial tendrán un efecto relevante en la retórica y en la práctica de las empresas turísticas, ya que intervienen en la representación del contexto estratégico (apartado 3.1), en la definición de los objetivos de las estrategias (3.2) y en su justificación como prácticas legítimas (3.3). Antes de presentar los resultados de la investigación será preciso exponer la perspectiva teórica neoweberiana desde la que adquieren relevancia los elementos socioculturales en la acción estratégica empresarial (Boltanski y Chiapello, 2002; Sennett, 2008), así como la estrategia metodológica seguida para acceder a este tipo de fenómenos.

\section{Marco teórico y metodológico}

La teoría económica neoclásica describe la cuestión que hemos planteado como un problema de ajuste a largo plazo de la función de producción. En función del desplazamiento previsto en la demanda se optará por una combinación de inputs (calidad) u otra (costes) según la rentabilidad que ofrezcan (Samuelson y Nordhaus, 2003). Sin embargo, aunque los empresarios pudieran disponer de información fiable sobre el estado futuro de estos parámetros, y superaran sus limitaciones cognitivas a la hora de tomar decisiones complejas (Bettman et al., 1998; Kahneman y Tversky, 1979; Tversky y Kahneman, 1974), quedaría por resolver el problema de qué decisión tomarían si identificaran dos soluciones con iguales expectativas de rentabilidad, una orientada a la calidad y otra a los costes.

1 Esta investigación fue coordinada por el autor en el marco de un Convenio de Colaboración entre el IESACSIC y la Consejería de Turismo, Comercio y Deporte de la Junta de Andalucía. Los datos primarios y los informes de investigación de dicho proyecto pueden solicitarse al Banco de Datos del IESA-CSIC. Para una exposición detallada de los planteamientos teóricos y los resultados de la investigación, consultar la tesis doctoral del autor (Rodríguez González, 2011). 
Es decir, ¿qué ocurre si la regla de maximización de los beneficios no permite discriminar entre dos estrategias alternativas? ¿Recurrirán los empresarios a algún criterio extraeconómico para decidir?

Desde Max Weber y su trabajo sobre el espíritu del capitalismo (Weber, 1905), la sociología ha planteado la existencia de valores extraeconómicos en los roles empresariales y gerenciales. Con posterioridad, Bendix (1956) retomó la temática marxista de la ideología capitalista atendiendo al componente ideológico del discurso de gestión (o managerial), apuntando a su influencia en el éxito de las estrategias empresariales; señaló que las estrategias, detrás de la búsqueda racional de eficacia, esconden un trabajo retórico de legitimación de la autoridad sobre el proceso productivo. Encontramos aquí una primera llamada de atención sobre el discurso de los empresarios y directivos en tono de sospecha, atribuyéndole un carácter ideológico (Chiapello, 2003), que después será retomado por las investigaciones posestructuralistas centradas en las dimensiones culturales y simbólicas de las estrategias empresariales.

Los enfoques neoinstitucionalistas mantienen un diálogo más cercano con la ortodoxia económica. A medida que se ha ido incorporando al mainstream la idea de que existe una esfera institucional que regula o constriñe el comportamiento en los mercados, los elementos que se incluyen en dicha esfera han ido aumentando. Si los economistas neoinstitucionalistas se centraron en el efecto de la coordinación jerárquica y las regulaciones legales en los costes de transacción del mercado (North, 1991; Williamson, 1981), los sociólogos neoinstitucionalistas añadieron el nivel intermedio del campo organizacional (DiMaggio, 1981), en el que las redes de relaciones interpersonales (Granovetter, 1985; 2005) y los marcos culturales compartidos (DiMaggio y Powell, 1983; Meyer y Rowan, 1977) en el seno de una industria dan lugar a patrones de conducta (isomorfismo) y estructuras de gobernanza que solucionan los problemas de confianza e incertidumbre en las relaciones económicas. Respecto al tema que aquí nos ocupa, resulta particularmente interesante la contribución de Meyer y Rowan (1977). Detrás de su provocadora idea de que parte del comportamiento organizacional es ceremonial, encontramos la fructífera tesis de que la legitimación ante otros actores del contexto organizacional puede ser un determinante tanto o más poderoso de las estrategias empresariales que la búsqueda de eficacia organizativa. Los estrategas empresariales pueden adoptar una determinada estrategia porque ésta sea una señal de confianza que induzca la lealtad de los inversores, trabajadores o clientes, aunque suponga una merma de la eficacia o la rentabilidad. La idea clave es que la eficacia de una estrategia es construida en conexión con las necesidades de legitimación de la organización en su contexto organizativo, en el que actores 
internos y externos que controlan recursos necesarios comparten o confrontan ideas mitificadas de lo que debe ser el funcionamiento de la organización, sus objetivos y sus parámetros de eficacia.

Todo este esfuerzo por poner de manifiesto la existencia de condicionantes sociales en las estrategias empresariales se desarrolla en paralelo al avance de las ciencias de la administración - o el Management-, un conjunto de tecnologías, prototecnologías y pseudotecnologías (Bunge, 1985) de la organización que, desde Taylor hasta Moss-Kanter, han construido un voluminoso cuerpo de conocimiento codificado dirigido a orientar el comportamiento de los directivos, proporcionándoles las herramientas cognitivas con las que confeccionar sus estrategias. En tanto que este conocimiento que describe el mundo de las organizaciones también se emplea para actuar en dicho mundo, nos encontramos con un conocimiento de carácter performativo, que crea el mundo que pretende describir (Callon et alii, 2002; Lamo de Espinosa, 2004; MacKenzie, 2006).

Varios autores han continuado las indagaciones de Bendix sobre la ideología empresarial acercándose con una perspectiva crítica al corpus del Management. Algunos investigadores han puesto de manifiesto las similitudes entre la sucesión de escuelas y gurús del Management y las modas (Abrahamson, 1996). Otros han puesto de relieve cómo, junto a los contenidos técnicos y altamente racionalizados, poseen una importancia crucial los contenidos normativos y justificativos, particularmente los encaminados a dotar a los empresarios y directivos de una retórica con la que movilizar y obtener la colaboración de los actores en los procesos de cambio organizacional (Gómez Rodríguez y López-Aranguren, 2004). Con un enfoque heredero de las ideas de Bourdieu sobre el pensamiento económico (Bourdieu, 2003), otros autores han puesto de relieve la variabilidad histórica de este género de discursos de gestión apuntando también algunas regularidades estructurales. Así, Alonso y Fernández-Rodríguez han señalado que la gestión del componente humano dentro de la organización es el tema central de la literatura de gestión, que «durante los últimos 40 años [...] ha reflejado las transformaciones del sistema productivo, escenificando, ejemplarizando y prescribiendo la transición de un modelo de producción en serie realizado en un ámbito normativo de seguridad, a uno de producción flexible incrustado en todas las convenciones posibles de individualización, incertidumbre y riesgo» (Alonso y Fernández-Rodríguez, 2006: 129).

Los efectos de este paso de un capitalismo sólido a un capitalismo líquido en las convenciones sociales que manejan los ejecutivos también ocupa una parte importante de la obra de Boltanski y Chiapello, El nuevo espíritu del capitalismo (2002), que rescata desde una perspectiva estructuralista genética la preocupa- 
ción weberiana por el entramado moral que sostiene las disposiciones a la acción que son imprescindibles para el funcionamiento del sistema capitalista. Estos autores identifican distintos déficits de legitimidad en el corpus teórico-filosófico del capitalismo, que son solventados mediante las justificaciones de estatus que articula el discurso de gestión (ver también Sennett, 2008). Estos dispositivos culturales, histórica y localmente contingentes, son claves para la reproducción del sistema capitalista, ya que incorporan criterios morales que justifican los arreglos y cambios en los procedimientos organizativos que requiere el momento epocal del capitalismo. Simplificando su modelo, nos interesa retener su idea de que el imperativo empresarial de la obtención de beneficios económicos es disfrazado ideológicamente en las sucesivas condiciones sociotécnicas y sociopolíticas, vinculándolo con una serie de beneficios sociales (desarrollo e innovación, libertad, seguridad) y recurriendo a valores morales no económicos (las «ciudades»: audacia, autoridad familiar, inspiración, meritocracia, etc.) que legitiman la acción empresarial dotándola de un sentido moral más amplio que la búsqueda egoísta de riquezas.

A partir de estas reflexiones teóricas, el objetivo de la investigación antes formulado se encamina a examinar los cambios en el discurso legitimador de los directivos y empresarios turísticos andaluces en el contexto del cambio de modelo turístico. Este discurso se produce desde el marco cultural de significados y creencias acerca del mundo de las empresas y los mercados que, a partir de distintos mecanismos de incorporación cultural (Merton y Wolfe, 1995), da lugar a un conocimiento de sentido común (Chamorro, 2006) sobre la organización y la gestión de las empresas, vinculado y conectado con los productos genuinamente científicos, pero pasado por el tamiz de la experiencia local e históricamente determinada de los grupos de empresarios y directivos que lo manejan. Apoyándonos en la distinción planteada por Fernández Rodríguez (2007), nuestro enfoque implica pasar del análisis de la producción de ideas de gestión - la literatura de management, a la que atienden Boltanski y Chiapello (2002) o Alonso y Fernández Rodríguez (2006) - al análisis de su consumo, atendiendo al uso que hacen de ellas los directivos y empresarios en contextos concretos.

Por otra parte, a partir de las investigaciones precedentes sobre el sector turístico español, es posible derivar algunas cuestiones relevantes para el planteamiento de la investigación. En primer lugar, las especificidades de la producción turística, compuesta por un agregado de actividades geográficamente localizadas y definidas por su clientela, aconsejan tomar como unidad de análisis el destino o localidad turística, entendido como un sistema de producción local que, a la manera de los distritos industriales, combina relaciones de competencia y colaboración 
y acarrea una importante repercusión en distintos aspectos del comportamiento de las empresas (Castellanos y Pedreño, 2006; Marrero Rodríguez, 2004; Santana Turégano, 2004). En segundo lugar, estas investigaciones han puesto de manifiesto la centralidad de los problemas de gestión del personal en el ámbito de la reestructuración turística. A grandes rasgos, cabe sintetizar este «problema del empleo turístico» como la tensión entre una retórica de la calidad y el compromiso profesional y unas prácticas orientadas a la flexibilidad, con consecuencias precarizantes (Ciria Navas, 2008). En este sentido, la investigación, por ejemplo, de Brunet y Alarcón (2007), pone de manifiesto que la mejora en el control sobre los trabajadores (y no de los ingresos o la rentabilidad) constituye el efecto más destacable de las estrategias de certificación de calidad que aprecian los directivos hoteleros catalanes que las adoptan. Como argumentan los autores, esto es debido a que la estandarización de los procedimientos mediante manuales de operaciones que requieren estas estrategias permite reducir los costes de la intensa rotación de personal que sufren estas empresas, a la vez que proporciona importantes instrumentos de control a los supervisores.

\subsection{Aspectos metodológicos}

De lo expuesto hasta ahora se sigue que el objeto de nuestra investigación es la cultura empresarial y, dentro de ella, los discursos estratégicos empresariales, entendidos como ideología en una doble acepción: como productos verbales que reflejan pensamientos socialmente condicionados (la noción de Mannheim, más próxima a lo que hoy se entiende como cultura) y como realizaciones prácticas del orden simbólico con el que los grupos poderosos amoldan a sus intereses las formas de representar el mundo y la acción en él (más en la línea de la noción marxista de pensamiento distorsionado o mitificador).

La técnica de investigación elegida para acceder a estos objetos culturales ha sido el Grupo de Discusión (en adelante, GD). Para justificar esta decisión, y en lo tocante a la ejecución práctica de la técnica, nos hemos apoyado en las reflexiones de Martín Criado (1997). Este autor, descontento con la impronta psicoanalítica y althusseriana que Jesús Ibáñez (1979) dio a las bases teóricas del GD, desarrolla una explicación sociológica de las causas por las cuales el GD es capaz de generar discursos colectivos y de las condiciones en que debe ejecutarse esta técnica para que sea efectiva. Su explicación parte de las ideas de Goffman sobre las estructuras sociales de la interacción (Goffman, 1974) y de los planteamientos de Bourdieu sobre la economía política del lenguaje (Bourdieu, 1985). A grandes rasgos, el argumento de Martín Criado viene a ser que el GD es eficaz 
en la medida en que sea capaz de crear, artificialmente, una situación en la que a los participantes no les quede otro remedio que utilizar el marco de sentido (el frame de Goffman) del colectivo social que da identidad al grupo (dado el anonimato entre los asistentes y la obligación de hablar sobre un tema). Así mismo, las sucesivas bazas con las que los participantes negocian su estatus dentro del grupo (siguiendo las reglas de la economía comunicativa de Bourdieu) recurren a los objetos y posiciones discursivas con mayor legitimidad para el colectivo de referencia. Buscando aproximarnos a esta situación ideal, en el reclutamiento y la ejecución de los GD se aplicaron las recomendaciones de Martín Criado sobre el anonimato y homogeneidad de los participantes, la intervención mínima del moderador y la búsqueda de neutralidad e informalidad en la configuración de los referentes materiales y simbólicos de la situación.

La variable dependiente de nuestra investigación es el discurso gerencial sobre el dilema entre calidad y costes en el contexto de la reestructuración turística. Las variables independientes se recogieron controlando la ubicación y la composición de los seis GD realizados. El diseño atendió al tipo de destino turístico (litoral, urbano o rural) y su nivel de desarrollo (maduro o emergente), al tipo de establecimiento (cadenas hoteleras, alojamientos independientes, oferta complementaria) en que trabajaban los participantes, así como a la función gerencial que desempeñaban (empresarios, gerentes y altos directivos, mandos intermedios). En la Tabla 1 se recogen los detalles de cada uno de los grupos realizados.

Tabla 1 Diseño de los Grupos de Discusión

\begin{tabular}{|c|c|c|c|c|c|}
\hline Código & Localidad & $\begin{array}{c}\text { Posición } \\
\text { jerárquica }\end{array}$ & Tipo de oferta & $\begin{array}{c}\text { Orientación } \\
\text { temática }\end{array}$ & $\begin{array}{c}\text { Grado de } \\
\text { desarrollo }\end{array}$ \\
\hline GD1 & Sevilla & Directivos & Alojamiento & Urbano & Maduro \\
\hline GD2 & Marbella & $\begin{array}{c}\text { Mandos } \\
\text { intermedios }\end{array}$ & Alojamiento & Litoral & Maduro \\
\hline GD3 & $\begin{array}{c}\text { Roquetas de } \\
\text { Mar }\end{array}$ & Directivos & Alojamiento & Litoral & Incipiente \\
\hline GD4 & Ronda & $\begin{array}{c}\text { Pequeños } \\
\text { empresarios }\end{array}$ & Mixto & $\begin{array}{c}\text { Urbano - } \\
\text { Rural }\end{array}$ & Incipiente \\
\hline GD5 & $\begin{array}{c}\text { El Rocío }- \\
\text { Matalascañas }\end{array}$ & $\begin{array}{c}\text { Pequeños } \\
\text { empresarios }\end{array}$ & $\begin{array}{c}\text { Actividades } \\
\text { complementarias }\end{array}$ & $\begin{array}{c}\text { Litoral }- \\
\text { Rural }\end{array}$ & Incipiente \\
\hline GD6 & Benalmádena & Gerentes & Alojamiento & Litoral & Maduro \\
\hline
\end{tabular}

Las transcripciones de los GD fueron analizadas con Atlas.ti 5.0. Dicho programa, aunque inspirado por los planteamientos metodológicos de la teoría fundamentada (Glaser y Strauss, 1967; Trinidad et alii, 2006), incorpora un conjunto de herramientas que permiten automatizar algunos de los procedimientos 
comunes al análisis de discursos, cualquiera que sea la perspectiva adoptada (Ruíz Ruíz, 2009; Valles Martínez, 2001). En nuestra investigación, el análisis se dirigió al desarrollo de modelos conceptuales o redes semánticas que dieran cuenta de las regularidades discursivas en torno al proceso de elaboración de estrategias. Para ello, se realizó un trabajo de codificación selectiva que buscaba la saturación teórica de los discursos recogidos, partiendo de un conjunto de códigos a priori extraídos de la literatura sobre la reestructuración turística (Agarwal, 2002; Hjalager, 2007; Ioannides y Debbage, 1997). Con posterioridad, se establecieron las relaciones entre los distintos códigos partiendo de la secuencia estándar del proceso estratégico (Chafee, 1985), aunque con intenciones netamente sociológicas: 1) el diagnóstico del contexto - formas de representación del mercado como sistema complejo-, 2) la identificación de estrategias alternativas y sus consecuencias - las teorías o modelos de gestión - y 3) la elección de la estrategia a implementar - la justificación o legitimación de las decisiones extraeconómicas en la selección de estrategias-.

\section{Resultados de la investigación}

\subsection{El diagnóstico del contexto}

Abordaremos aquí los elementos que utilizaron los empresarios y directivos para construir, en el transcurso de los GD, su representación del mundo del mercado turístico. Obviamente, no encontramos en estos discursos sobre el ambiente o entorno en el que las empresas deben «sobrevivir»y «adaptarse» nada similar a la información perfecta que los modelos microeconómicos neoclásicos esperan de los actores económicos por excelencia. En su lugar, emerge una visión coral de la situación del mercado turístico como un sistema complejo, sobre el que existen tanto certezas como incertidumbres. En dicha visión, y junto a las comprensibles diferencias de perspectiva, encontramos también notables regularidades. Quizás la más relevante sea la oposición entre viejo y nuevo turismo, un recurso expositivo omnipresente en la totalidad de los grupos que, incorporando al sentido común empresarial la noción académica de la caducidad del modelo turístico masivo, apunta a una clara ruptura entre el modelo de desarrollo turístico español de las décadas de 1960 a 1980 y la situación actual. Al igual que ocurre en la oferta académica de ideas de gestión, las descripciones del «viejo» turismo resultan más nítidas y unánimes que las del «nuevo» turismo, que, precisamente por su novedad, plantean más dificultades a los participantes en la investigación. Con las debidas precauciones, la visión del viejo turismo conlleva muy frecuentemente la 
connotación de lo estable y predecible, mientras que el nuevo turismo lleva casi siempre asociada la incertidumbre y la inseguridad.

$\mathrm{H}$ : Ha habido cambios muy radicales en los últimos cinco años. Realmente, un hotelero antes, la forma de gestionar un hotel, a nivel comercial, no es que fuera cómoda, pero bueno, era decir, yo tengo 100 habitaciones, pues voy a contratar 180 o 180, lo que se llamaba técnicamente over-contracting, ¿no? [... ]

$\mathrm{H}$ : Tenías tú la sartén ahí.

H: Tú efectivamente te tenías que enfadar con alguno, y decías, «oye, que yo tengo un cupo», «sí, pero tú ayer no me lo vendiste» [... Con lo cual siempre hay una pelea un poquito equilibrada, sana. $\mathrm{Y}$ lo que era bueno para nosotros, es que realmente faltaban camas. Faltaban camas, porque yo [...] [viví] un poquito la época de muchísimo trabajar, pero una época que añoro. Es decir, señores, no era tan difícil llevar un hotel al fin y al cabo: era introducirle turoperadores. (GD6)

No obstante, la oposición entre viejo y nuevo turismo sigue líneas muy diferentes según los diferentes elementos que dan lugar a la otra gran regularidad del discurso sobre el contexto: la parcelación del mercado en un conjunto relativamente reducido de actores colectivos, y el reconocimiento de relaciones e intercambios con ellos que trascienden de forma relevante la esfera económica. Estos actores son: los turistas, la competencia, los destinos turísticos, las administraciones públicas y los distribuidores e intermediarios.

Es posible realizar una lectura transversal de los distintos «dilemas» que se plantean a la hora de enjuiciar a los objetos del contexto estratégico, sus propiedades y relaciones. Podemos identificar dos temas de mayor abstracción teórica que vertebran las distintas percepciones sobre el estado de la cadena de valor. En primer lugar, existe un problema respecto a las especificaciones del producto, que se deriva de los cambios en las expectativas y exigencias de los turistas, la multiplicación de productos singulares de la competencia, la responsabilidad limitada de los empresarios en el producto agregado del destino turístico y la erosión del papel de los intermediarios en la definición de los estándares que favorecen las nuevas tecnologías. Como resultado de todos estos procesos, se desdibujan los contornos que delimitaban los componentes específicos de la mercancía turística, en cuanto agregado complejo de productos y servicios. En los apartados siguientes veremos que a este problema le corresponde una serie de estrategias (la mejora de la calidad) que se justifican en relación a una consideración de tipo extraeconómico (la ética de la profesionalidad).

En segundo lugar, es posible plantear que existe una mayor incertidumbre respecto a los precios, que afecta de forma importante a los distintos juicios sobre los 
elementos del contexto. Esta preocupación por los precios se manifiesta de forma clara en los pronunciamientos sobre el crecimiento de la oferta y la necesidad de afrontar la entrada de nuevos competidores con estructuras de costes menos gravosas, así como en el debate acerca de los nuevos intermediarios y la elevada flexibilidad de los precios en los actuales canales comerciales. Pero también está presente de forma significativa en la percepción instrumental del turista y las tensiones entre precio y nivel de exigencia que caracterizan las interacciones con el cliente final. Es posible, así mismo, rastrear esta preocupación en los posicionamientos sobre la intervención de las administraciones públicas en los mercados para reforzar la reputación de los destinos turísticos y sus infraestructuras de transporte. Hemos visto que, por distintas vías, las consideraciones respecto al precio ganan preponderancia sobre las consideraciones acerca de las especificaciones del producto, de tal forma que, cada vez más, el problema del empresario no es a qué precio vender un producto respecto a unos determinados costes de elaboración, sino qué costes de elaboración puede asumir para vender a un precio determinado. Frente a la hipótesis económica de los mercados eficientes, según la cual «los precios de los activos reflejan permanentemente toda la información disponible y, en ese sentido, la valoración de mercado es correcta» (Zurita, 2009: 183), los empresarios de nuestra investigación se quejan de que el precio no contiene toda la información relevante y que, de hecho, es un indicador cada vez menos eficiente de las cualidades del producto. Como veremos en los próximos capítulos, al igual que con las presiones sobre las especificaciones del producto, la incertidumbre respecto a los precios es un rasgo del contexto que se corresponde con una respuesta estratégica específica (la flexibilización y racionalización de procesos) y adopta un dispositivo justificativo particular (la justificación moral de la rentabilidad empresarial como fuente de bienestar colectivo).

M: Hay dos tipos de clientes: el cliente de verdad que paga la habitación, incluso fuera de Semana Santa, a un precio muy alto porque son las últimas que te quedan, y las pongo a tal precio y las vendo, estupendo. Y el cliente que reserva a través de internet, que ven el precio, que no pueden decir que le hemos dicho otro precio, que lo aceptan y aceptan las condiciones, y que luego se quejan de que es caro. (GD1)

\subsection{Las estrategias como tecnologías de gestión}

Acabamos de plantear la idea de que dos rasgos del entorno - la flexibilidad de precios y la indeterminación de las especificaciones del producto- eran afron- 
tados con dos orientaciones estratégicas - flexibilización y calidad- que se enraizaban en construcciones normativo-culturales - la rentabilidad y la profesionalidad-.

A la hora de analizar el discurso sobre las estrategias recogido en los GD se buscó identificar los elementos básicos de la teoría de gestión que subyace en ambas orientaciones estratégicas. En este análisis, junto a algunos temas clave del Management académico (calidad total, downsizing y externalización, tecnofilia), encontramos construcciones discursivas firmemente ancladas en la experiencia local de los empresarios dentro del campo organizacional turístico. Dichas construcciones, independientemente de su mayor o menor validez en cuanto tecnologías de gestión, incorporan de forma más o menos evidente una proyección de los intereses del grupo a las formas de operar sobre las organizaciones empresariales y sus componentes.

Este resultado viene a confirmar una idea ya apuntada en el Apartado 2: que el discurso estratégico, en cuanto lenguaje para representar la acción, tiende a prefigurar sus resultados, de forma que la representación, hasta cierto punto, constituye (o da forma) lo representado. Quizás la evidencia más clara en este sentido sea el heurístico que, en las estrategias de reorganización interna orientadas a la flexibilización, convierte un problema de eficiencia - las fluctuaciones de la demanda hacen que, en determinados momentos, algunos inputs sean relativamente improductivos - en un problema contable - hay que ajustar, en todo momento, los costes a los ingresos - y, de ahí, en un problema laboral - hay que limitar los compromisos a largo plazo con los trabajadores-. El énfasis en la calculabilidad introduce un sesgo cognitivo en la representación del «ambiente interno» de la empresa análogo al que se produce al reducir las cualidades de los clientes y competidores (el «ambiente externo») a sus precios.

$\mathrm{H}:[. .$.$] Hay una pequeña pescadilla que se muerde la cola, en el sentido de que$ queremos sostenibilidad y queremos dar buen servicio, pero las circunstancias no nos ayudan para ello. Evidentemente, una empresa para aportar calidad tiene que dar beneficio, beneficio suficiente para reinvertir parte de los ingresos en el mismo producto, e incluso, a veces, en el mismo capital humano, ¿no? (GD6)

Al desarrollar los mapas semánticos sobre las intervenciones acerca de las estrategias, hemos constatado que la reestructuración turística adopta de forma preferente determinadas pautas, de manera que se produce una limitación del repertorio estratégico a un conjunto limitado de prácticas. La rentabilización a corto plazo de las inversiones, la racionalización y tecnificación de los procesos, la 
flexibilización del trabajo y la conformidad respecto a los estándares del producto del campo organizacional aparecen como estrategias preferentes (o, incluso, inevitables) en la caracterización del plan estratégico de la empresa. En contraste, las estrategias del repertorio «recalificador» de la reestructuración, encaminadas a la diferenciación del producto, el conocimiento profundo de la demanda o la generación de economías del conocimiento a partir del capital humano, tienen mucha menos presencia en el discurso empresarial y, de hecho, aparecen fundamentalmente en los grupos situados en los márgenes del campo organizacional (mandos intermedios del litoral, empresarios de oferta complementaria rural).

$\mathrm{H}:[\ldots]$ Yo he recomendado restaurantes que a lo mejor hace dos años tenían un estándar de calidad bastante aceptable, una relación calidad-precio muy buena, y ahora han bajado un poquito los precios porque... Yo lo veo normal: tienes un personal, unos impuestos que tienes que cubrir. $\mathrm{Y}$, o trabajas con cuatro clientes a precio muy alto, lo cual va a ser muy difícil cubrir gastos, o trabajas con muchos clientes a un precio más bien bajo. Con lo cual tienes que bajar los estándares de calidad, en cuanto a restauración. (GD5)

En el plano teórico, este resultado viene a confirmar la idea neoinstitucionalista de la difusión de estrategias, que apunta a la existencia de conjuntos restringidos de estrategias legítimas con mayor probabilidad de difusión, elaborados colectivamente dentro del campo organizacional y en los que la eficiencia económica no es el único criterio que justifica la presencia de una estrategia. Con respecto al problema sustantivo de la reestructuración turística, las evidencias recogidas vienen a indicar que, de las dos caras de la pauta empresarial posfordista (la especialización flexible), los empresarios turísticos andaluces están predispuestos a la flexibilidad pero poco inclinados a la especialización. Tomadas en conjunto, estas estrategias parecen más orientadas hacia el liderazgo en costes que hacia la diferenciación competitiva (Porter, 1985).

Este diagnóstico pesimista sobre la posibilidad de la recalificación se ve reafirmado por el «problema del empleo». Las consecuencias laborales de la racionalización y flexibilización de procesos conducen a una quiebra del sistema que producía las habilidades profesionales de los trabajadores (Brunet y Belzunegui, 2003) en el «viejo» turismo. Es de destacar aquí que nuestros resultados son muy similares a los obtenidos por investigaciones precedentes: los empresarios no dan tanta importancia a las competencias técnicas del profesional turístico (ya se adquieran éstas a través de la escuela o de la experiencia) como a sus competencias sociales (la servicialidad) y su compromiso con la empresa (Castellanos y Pedreño, 2006; Marrero Rodríguez, 2004; Marrero Rodríguez, 2007). 
El desmantelamiento de los canales paternalistas de socialización laboral y promoción profesional que produce la desregulación laboral (Alonso, 2001) tiene como consecuencia no deseada la aparición de dificultades para inculcar a los trabajadores una nueva profesionalidad («ser profesionales de otra forma») en las condiciones de flexibilidad estructural que introducen los nuevos arreglos organizativos. Esta nueva profesionalidad busca sustituir el compromiso con la empresa por un compromiso con la profesión, que mantenga a los trabajadores flexibles dentro del mercado laboral del sistema de producción local. En una manifestación claramente ideológica, los empresarios atribuyen el escaso éxito de estos planteamientos a, entre otros aspectos, el excesivo materialismo de los trabajadores - que al igual que los competidores ilegítimos, no buscan realizarse profesionalmente, sino enriquecerse, y no entienden las presiones competitivas del mercado- y al escaso apoyo institucional del turismo en comparación con la actividad inmobiliaria. Cualquier excusa es buena salvo reconocer — como hace el grupo marginal de mando intermedios- que quizás exista un problema en la relación entre el tipo de trabajo que se demanda - las condiciones laborales de los empleos- y el precio al que se paga — los salarios y los derechos sociales-. $\mathrm{El}$ hecho de que en este nuevo régimen laboral las competencias profesionales no se adquieran en la empresa, sino mediante la rotación entre empresas, nos lleva a plantear, como conjetura, que los trabajadores tendrán pocos incentivos para especializarse profesionalmente más allá de los perfiles medios — los estándares de atención y trato- del destino turístico, lo que situará a las empresas en dificultades a la hora de obtener el componente humano necesario para sus estrategias de calidad. Es decir, que una consecuencia no deseada de las estrategias de flexibilización es la erosión de los mecanismos de reproducción del contingente laboral turístico, tanto en términos cuantitativos - la población local ya no quiere trabajar en el turismo, hay que traer inmigrantes - como en términos cualitativos — los nuevos trabajadores turísticos no son profesionales-.

$\mathrm{H}$ : Eso es muy complicado, porque depende de las necesidades y del momento. Hoy se nos va un recepcionista y tenemos que buscar uno ya, para mañana, pues prácticamente... prácticamente no, francamente, casi el primero que se presente o esté disponible es al que echas mano. Después lo tendrás el tiempo que puedas tenerlo, hasta que busques a otro cualificado, si lo encuentras, si no tienes que aguantar con él y tienes que estar todo el día pendiente de él. Y la jefa de recepción pues tiene que estar todo el día pendiente de fulanito para que le haga las cosas bien hechas y atienda como Dios manda [...], en todas las empresas ocurre lo mismo, depende de las necesidades del momento. (GD4) 
Por último, el análisis de las estrategias orientadas hacia la calidad muestra que, si bien la profesionalidad (el compromiso con el producto) aparece como el dispositivo de justificación central, las estrategias empresariales consisten (o son descritas) como actividades de «observación» de estándares. Aquí, la «observación» alude tanto al examen de las cualidades de los productos de la competencia del campo organizacional regional como al cumplimiento de normas, formales e informales, sobre los requisitos mínimos que deben realizar los productos propios. En esta actividad de reconocimiento de un conjunto de propiedades, colectivamente establecidas, de los productos turísticos, se parte de un significado de la calidad que desvía y desplaza la repercusión de esta estrategia, liberando a los empresarios de la responsabilidad en su consecución. Este significado, que hemos adjetivado como calidad integral, entiende la calidad como un resultado colectivo que implica a todos los actores del destino - alojamiento, oferta complementaria, administraciones, trabajadores, intermediarios - y que, en la medida en que identifica comportamientos oportunistas en estos actores - competidores desleales, oferta complementaria no profesionalizada, administraciones no comprometidas con el desarrollo turístico, trabajadores materialistas, intermediarios comisionistas - y en los clientes - exigencias ilegítimas de los clientes de oferta-, permite que el discurso de la calidad «de puertas para fuera» actúe como justificación para el ajuste a la baja de los estándares de calidad y la adopción de estrategias tendentes a la macdonalización (Ritzer, 2002).

\subsection{Rentabilidad y calidad como fines legítimos de las estrategias}

Dirigimos, por último, nuestra atención hacia los valores que se manifiestan en las justificaciones de las estrategias. Examinamos, de este modo, los objetos culturales que actúan como elementos centrales del lenguaje de motivos con que se (auto) describe el comportamiento estratégico de los empresarios turísticos: creencias compartidas (y normas basadas en ellas) sobre lo correcto o incorrecto (bueno o malo) de los distintos objetos (sucesos, comportamientos) que intervienen en la decisión estratégica empresarial. Más concretamente, nos centraremos en los fines que se plantean en los objetivos de las estrategias y en los mecanismos discursivos mediante los cuales se establece su legitimidad (su aceptabilidad social). De esta forma, en la medida en que vamos a tratar la construcción de la legitimidad en las actividades económicas de los empresarios turísticos andaluces, nos aproximamos a una caracterización localizada del espíritu del capitalismo, tal y como lo conciben Boltanski y Chiapello (2002). 
Este análisis atiende a la presencia de criterios extraeconómicos en la elaboración de las estrategias. Los resultados de nuestra investigación muestran que, a la hora de definir y elegir una estrategia para su empresa, los empresarios no se orientan únicamente por la maximización de la rentabilidad, tal y como presumen los modelos económicos del comportamiento empresarial, sino que también tienen en cuenta otros criterios distintos, o incluso opuestos. No se quiere decir con ello que los empresarios turísticos describan su comportamiento como irracional, sino que lo describen como racional respecto a unos fines más amplios que el constante incremento de sus ganancias. Nuestro análisis apunta a que, de acuerdo con las funciones del espíritu del capitalismo que identifican a escala teórica Boltanski y Chiapello, este entramado moral juega un papel importante en el ajuste a medio y largo plazo del campo organizacional a los condicionantes de su entorno, limitando y reorientando sus prácticas de generación de beneficios.

En nuestro examen de las justificaciones de los empresarios sobre sus decisiones estratégicas poseen especial protagonismo las que hemos denominado racionalizaciones económicas. Se trata de argumentaciones en las que intervienen conceptos y creencias incorporados (en el sentido mertoniano a que hacíamos referencia en el apartado 2) desde la ciencia económica, dando lugar a fenómenos de performatividad genérica y efectiva. Estas justificaciones plantean las estrategias como la consecuencia del imperativo natural de la búsqueda de la rentabilidad: la empresa debe ser rentable para sobrevivir, está en su «naturaleza» adaptarse a las condiciones del mercado adoptando las prácticas que resultan competitivas para hacerse con una porción menguante de los beneficios, al menos desde la lectura del contexto estratégico a que hemos hecho referencia.

Este tipo de justificaciones viene, de forma típica, a eximir a los empresarios turísticos de cualquier responsabilidad en las estrategias de flexibilización de costes y sus repercusiones en el empleo («despides porque no te queda otro remedio»). Junto a esta visión de una rentabilidad amenazada por la ley de la selva del mercado se suma la afirmación de una serie de efectos derivados de la acción capitalista que, siguiendo el tema clásico (al menos desde Adam Smith) de los beneficios colectivos derivados de la persecución egoísta de riquezas, apunta a que los empresarios generan mayor eficiencia, innovan y contribuyen al bienestar colectivo. La pieza central de todo este discurso es que las empresas «crean empleo», vinculando de forma inevitablemente ideológica la producción capitalista de mercancías turísticas con el mantenimiento de la población en las localidades donde se asienta. 
H: [... Esto afecta a la rentabilidad, y cuando afecta a la rentabilidad afecta también al mantenimiento de los puestos de trabajo. (GD12)

Merece la pena abordar, aunque sea superficialmente, los rasgos ideológicos del discurso empresarial sobre el empleo turístico y su papel en la justificación de las estrategias. En el contexto de los problemas de reproducción del contingente laboral planteados en el apartado anterior, los empresarios y directivos establecen una distinción entre el ámbito laboral — sujeto a flexibilización y subordinado a la rentabilidad empresarial - y el ámbito profesional —orientado por la estandarización y dirigido a la calidad turística-. Se entiende —o al menos así se manifiesta al tratar, por ejemplo, las relaciones con los sindicatos - que el trabajador debe disociar sus derechos laborales - salario y condiciones de trabajo- de sus deberes profesionales — ser autoexigente y adaptativo-. Recuperando la vieja terminología marxista, separar el valor de cambio - el salario que fija el mercado- de la mercancía trabajo de su valor de uso - su contribución al proceso productivo-.

El discurso de la profesionalidad plantea otros rasgos aún más relevantes para el objeto de este apartado. Aparte de su papel retórico en la consecución del consentimiento en la producción (Burawoy, 1989), juega un papel esencial como contrapeso a la búsqueda de rentabilidad en la justificación de las estrategias. Es el ejemplo más claro en nuestros datos de un elemento de la cultura empresarial que «constriñe el proceso de acumulación» limitando sus excesos (Boltanski y Chiapello, 2002: 26). Sintetizando a grandes rasgos, la profesionalidad es el elemento crucial de la identidad de los empresarios turísticos que les lleva a no ser sólo empresarios, sino también profesionales turísticos. Esta identidad o autoimagen, construida a lo largo de la trayectoria laboral que lleva a los directivos hasta la cúspide jerárquica de las empresas turísticas, actúa así como un freno moral en la búsqueda de la rentabilidad: no todo está justificado a la hora de obtener dinero de los turistas, sino que existen unos estándares profesionales que es preciso respetar.

$\mathrm{H}$ : Yo veo el problema en el propietario. Porque no tiene la mentalidad de crear una empresa profesional a medio-largo plazo, no puede transmitir eso al trabajador. (GD11) 


\section{Conclusiones}

Tomados en conjunto, los resultados sustantivos de esta investigación vienen a poner de manifiesto que el proceso de elaboración de estrategias empresariales está firmemente incrustado en el sistema de relaciones políticas y culturales del campo organizacional, en este caso, los sistemas locales de producción turística. Nuestras evidencias apuntan a que, tanto el diagnóstico del contexto como la identificación de las alternativas estratégicas y la selección de estrategias, son procesos cognitivos que se ven afectados por los sesgos epistemológicos que dan lugar a conocimientos socialmente condicionados. Hemos comprobado que el marco discursivo con el que se representan los objetos del proceso de elaboración incorpora de forma parcial e interesada las tecnologías organizativas que ofrece el corpus de ideas de gestión, prefigurando los resultados de la reestructuración turística. En futuros trabajos será preciso indagar con mayor profundidad sobre las consecuencias no deseadas - por ejemplo, un «círculo vicioso» de deterioro de los productos turísticos por el ajuste a la baja de los estándares y la falta de incentivos a la profesionalización que introduce la precariedad - que pueden acarrear este tipo de fenómenos.

En el contexto de este número monográfico, el artículo muestra que los temas clásicos de la «vieja» sociología económica — en nuestro caso, la idea weberiana de que el comportamiento económico reposa sobre determinados patrones culturales - siguen siendo relevantes para comprender los fenómenos económicos actuales. Así mismo, permite apuntar que las vías de exploración de estos fenómenos de la 'nueva' sociología económica son mucho más amplias de lo que los representantes anglosajones de esta corriente plantean (Smelser y Swedberg, 2005). Sus esfuerzos por incorporar variables parsonianas que doten de realismo a las explicaciones basadas en el homo economicus, tienen - junto con sus méritos- el inconveniente de que ignoran los problemas epistemológicos y teóricos que se derivan de los avances en la comprensión científica y filosófica del lenguaje y el significado. La revolución sociolingüística que supone pasar de una concepción universalista y sintáctica a una visión relativista y pragmática del significado ha sacudido profundamente, para bien y para mal, a las ciencias sociales. La ciencia económica, con sus prejuicios cuantitativistas, ha permanecido ajena a estos problemas, dejando un amplio campo para un programa sociológico de indagación sobre los aspectos cualitativos y simbólicos del comportamiento económico en general y, en particular, del de los empresarios y directivos. En esta línea, la modesta aportación que pretende este trabajo es la de dar difusión a estos planteamientos entre la comunidad científica interesada en los, por rescatar la noción weberiana, fenómenos económicos socialmente condicionados. 


\section{Bibliografía}

Abrahamson, E. (1996). «Management fashion», Academy of Management Review, 21(1): 254-285.

Agarwal, S. (2002). «Restructuring seaside tourism: The Resort Lifecyle», Annals of Tourism Research, 29(1): 25-55.

Alonso, L. E. (2001). Trabajo y posmodernidad: el empleo débil. Madrid: Fundamentos.

Alonso, L. E. y Fernández Rodríguez, C. J. (2006). «El imaginario managerial: El discurso de la fluidez en la sociedad económica», Política y Sociedad, 43(2): 127-151.

Barke, M. y Towner, J. (2004). «Learning from experience? Progress towards a sustainable future for tourism in the Central and Eastern Andalucían littoral», en Bramwell, B. (2004). Coastal Mass Tourism: Diversification and Sustainable Development in Southern Europe. Clevedon (UK): Channel View Publications: $157-175$.

BeNDix, R. (1956). Work and authority in industry: managerial ideologies in the course of industrialization. New Brunswick (New Jersey): Transaction Publishers. 2001.

Bettman, J. R.; Luce, M. F. et alii (1998). «Constructive consumer choice processes», The Journal of Consumer Research, 25(3): 187-217.

Boltanski, L. y Chiapello, E. (2002). El nuevo espíritu del capitalismo. Madrid: Akal.

Bourdieu, P. (1985). ¿Qué significa hablar? Economía de los intercambios lingüisticos. Madrid: Akal.

Bourdieu, P. (2003). Las estructuras sociales de la economía. Barcelona: Anagrama. Brunet, I. y Alarcón, A. (2007). «Calidad y recursos humanos del sector hotelero en Cataluña», Revista Internacional de Sociología, 47: 73-98.

Brunet, I. y Belzunegui, A. (2003). Flexibilidad y formación. Una crítica sociológica al discurso de las competencias. Barcelona: Icaria.

Bunge, M. (1985). Seudociencia e ideología. Madrid: Alianza.

Burawoy, M. (1989). El consentimiento en la producción: los cambios del proceso productivo en el capitalismo monopolista. Madrid: Ministerio de Trabajo y Seguridad Social.

Butler, R. W. (1980), «The concept of a tourist area cycle of evolution - Implications for management of resources», Canadian Geographer, 24(1).

Callon, M.; Meadel, C. et alii (2002). «The economy of qualities», Economy and Society, 31: 194-217. 
Castellanos, M. L. y Pedreño, A. (2006). Los nuevos braceros del ocio. Sonrisas, cuerpos flexibles e identidad de empresa en el sector turístico. Madrid-Buenos Aires: Miño y Dávila.

Ciria Navas, R. (2008). Recualificación empresarial y condiciones laborales en el sector turístico andaluz. VI Congreso Internacional Asociación Mediterránea de Sociología del Turismo. Granada.

Chafee, E. E. (1985). «Three models of strategy», Academy of Management Review, 10: 89-98.

Chamorro, J. M. (2006). Lenguaje, mente y sociedad. Hacia una teoría materialista del sujeto. La Laguna: Servicio de Publicaciones Universidad de La Laguna.

Chiapello, E. (2003). «Reconciling the Two Principal Meanings of the Notion of Ideology: The Example of the Concept of the "Spirit of Capitalism"», European Journal of Social Theory, 6(2): 155-171.

DiMaggio, P. (1981). «Structural Analysis of Organizational Fields», Annual Review of Research in Organizations, 8: 385-408.

DiMaggio, P. y Powell, W. (1983). «The iron cage revisited: institutional isomorphism and collective rationality in organizational fields», American Sociological Review, 48: 147-160.

Fernández Rodríguez, C. J. (2007). El discurso del Management: tiempo y narración. Madrid: CIS-Siglo XXI.

Glaser, B. G. y Strauss, A. L. (1967). The discovery of grounded theory strategies for qualitative research. New York: Aldine ( $1^{\text {a }}$ ed.)

Goffman, E. (1974). Frame analysis : an essay on the organization of experience. New York: Harper \& Row.

Gómez Rodríguez, C. y López-Aranguren, E. (2004). La retórica del cambio en las organizaciones. Un análisis aplicado. Madrid: CIS-Siglo XXI.

Granovetter, M. (1985). «Economic action and social structure: The problem of embeddedness», American Journal of Sociology, 91: 481-510.

Granovetter, M. (2005). «Business groups and social organizations», en Smelser, N. J. y Swedberg, R. (2005). The Handbook of Economic Sociology. Princeton, Princeton University Press\$ 429-450.

HJalager, A.-M. (2007). "Stages in the economic globalization of tourism», Annals of Tourism Research, 34(2): 437-457.

IвÁñez, J. (1979). Más allá de la sociología: el grupo de discusión, teoría y crítica. Madrid: Siglo XXI (1 $1^{\text {a }}$ ed.)

Ioannides, D. y Debbage, K. (1997). «Post-Fordism and Flexibility: The travel industry polyglot», Tourism Management, 18(4). 
Kahneman, D. y Tversky, A. (1979). «Prospect theory: An analysis of decision under risk», Econometrics, 47: 263-291.

Lamo de Espinosa, E. (2004). «¿Para qué la ciencia social?», en Giner, S. (2004). Teoría sociológica moderna. Barcelona, Ariel: 25-42.

LASh, S. y URrY, J. (1998). Economías de signos y espacio. Sobre el capitalismo de la posorganización. Buenos Aires: Amorrortu.

MacCannell, D. (2003). El turista. Una nueva teoría de la clase ociosa. Madrid: Melusina.

MacKenzie, D. A. (2006). An engine, not a camera: how financial models shape markets. Cambridge: MIT Press.

Marrero Rodríguez, J. R. (2004). La estructura y dinámica de los mercados de trabajo en las actividades de servicios: el caso del sector turístico canario. Las Palmas de GC: Servicio de Publicaciones del Cabildo Insular de Gran Canaria.

Marrero Rodríguez, J. R. (2007). «Las competencias sociales en las actividades de servicios. Acerca del aprendizaje y gestión empresarial de las mismas», en Rodríguez González, P; Ciria Navas, R. y Moreira Gregori, P. (2007). Turismo y Sociedad en Andalucía. Sevilla, Junta de Andalucía. Consejería de Turismo, Comercio y Deporteł 135-156.

Martín Criado, E. (1997). «El grupo de discusión como situación social», Revista Española de Investigaciones Sociológicas, 79(3): 81-112.

Mastny, L. y Peterson, J. A. (2001). Traveling light: new paths for international tourism. Worldwatch Papers. Michigan, Worldwatch Institute. 159: 88 .

Merton, R. K. y Wolfe, A. (1995). «The cultural and social incorporation of sociological knowledge», The American Sociologist, 26(3): 15-39.

Meyer, J. W. y Rowan, B. (1977). «Institutionalized Organizations: Formal Structure as Myth and Ceremony», American Journal of Sociology, 83(2): 340-363.

Mintzberg, H. (1983). La naturaleza del trabajo directivo. Barcelona: Ariel.

Mintzberg, H. (1988). La estructuración de las organizaciones. Barcelona: Ariel ( $1^{\mathrm{a}} \mathrm{ed}$.)

North, D. C. (1991). «Institutions», Journal of Economic Perspectivas, 5: 97-112.

OMT (1997). Turismo panorama 2020: influencias, flujos direccionales y tendencias claves. Resumen ejecutivo: Organización Mundial del Turismo.

Porter, M. E. (1985). Estrategia competitiva: Técnicas para el análisis de los sectores industriales $y$ de la competencia. México: Compaña Editorial Continental. $2^{a}$ impresión.

Ritzer, G. (2002). La McDonalización de la sociedad: un análisis de la racionalización en la vida cotidiana. Barcelona: Ariel ( $1^{\text {a }}$ ed.) 
Rodríguez González, P. (2011). La elaboración de estrategias empresariales en el sector turístico andaluz. Prácticas y discursos ante la reconversión turística. Departamento de Sociología. La Laguna, Universidad de La Laguna 476.

Ruíz Ruíz, J. (2009) «Análisis sociológico del discursoः métodos y lógicas», en Forum Qualitative Sozialforschung / Forum: Qualitative Social Research, vol. 71.

Samuelson, P. A. y Nordhaus, W. D. (2003). Economía. Madrid: McGrawHill (17 ${ }^{\mathrm{a}}$ ed.).

Santana Turégano, M. A. (2004). Formas de desarrollo turístico, redes y situación de empleo. El caso de Maspalomas (Gran Canaria). Barcelona, Universidad Autónoma de Barcelona.

Sennett, R. (2008). La cultura del nuevo capitalismo. Barcelona: Anagrama ( $3^{\mathrm{a}}$ ed.)

Smelser, N. J. y Swedberg, R. (2005). «Introducing Economic Sociology», en Smelser, N. J.y Swedberg, R. (2005). The Handbook of Economic Sociology, Second Edition. Princeton, Princeton University Press $\$ 3-25$.

Trinidad, A.; Carrero, V. et alii (2006). Teoría fundamentada «Grounded Theory». La construcción de la teoría a través del análisis interpretacional. Madrid: CIS.

Tversky, A. y Kahneman, D. (1974)+ «Judgment under uncertaintyः Heuristics and biases», en Science, 185(4157): 1124-1131.

URRY, J. (2002). The tourist gaze: leisure and travel in contemporary societies (2nd edition). London: SAGE.

Valles Martínez, M. S. (2001). «Ventajas y desafíos del uso de programas informáticos (p.e.ATLAS.ti y MAXqda) en el análisis cualitativo. Una reflexión metodológica desde la grounded theory y el contexto de la investigación social española», en Seminario sobre Investigación Avanzada Cualitativa Asistida por Ordenador, Granada, Fundación Centro de Estudios Andaluces.

Weber, M. (1905). La ética protestante y el espiritu del capitalismo. Barcelona: Península (1992).

Williamson, O. E. (1981). "The economics of organization: the transaction cost approach», American Journal of Sociology, 87(3):548-577.

Zurita, F. (2009). «Simposio Crisis global y teoría económica: la economía financiera frente a la crisis», Cuadernos de Economía, 46: 183-195. 